\title{
Salmonella Breast Abscess and Salmonella Mastitis: A Review and Update of the Literature
}

Anthony Kodzo-Grey Venyo ${ }^{1 *}$ Olawumi Adaramodu ${ }^{2}$

${ }^{1}$ North Manchester General Hospital, Delaunays Road Crumpsall Manchester, M8 5RB, United Kingdom

${ }^{2}$ Salford Primary Care Trust (EPIC Extended Primary Integrated Care), City Approach Eccles, Salford, Manchester, Lancashire, M30 OBL United Kingdom.

*Corresponding Author: Anthony Kodzo-Grey Venyo. North Manchester General Hospital, Department Of Urology, Delaunays Road, Manchester, M8 5RB. United Kingdom.

Received date: March 22, 2021; Accepted date: April 05, 2021; Published date: April 08,2021

Citation: Anthony K-G Venyo, O Adaramodu. (2021) Salmonella Breast Abscess and Salmonella Mastitis: A Review and Update of the Literature. Clinical Research and Clinical Trials. 3(2); DOI: 10.31579/2693-4779/031

Copyright: () 2021 Anthony Kodzo-Grey Venyo, This is an open access article distributed under the Creative Commons Attribution License, which permits unrestricted use, distribution, and reproduction in any medium, provided the original work is properly cited.

\begin{abstract}
:
Salmonella breast abscess / mastitis is a rare infection which can affect children and adults. It can affect females more commonly who are non-lactating and it does affect males as well as individuals who have predisposed conditions including: the immunocompromised, diabetics, rheumatoid arthritis, malignancies and other diseases. It may be acute, chronic, unilateral, or bi-lateral, as well as it may be recurrent if it is not appropriately treated. There tends to be a history of previous enteric fever (abdominal pain, vomiting, diarrhoea) which may not have been treated or adequately treated but this history has tended not to be apparent at the time of presentation. Manifestations of the disease tend to include a short- or long-history of: lump/mass in the breast which may or may not be painful, a history of previous diarrhoea, weight loss. The breast examination findings tend to be non-specific with some of the findings including tenderness or no tenderness, a mass that may or may not be tender, a fluctuant lump that may feel like containing fluid, a firm or hard feeling lump that may mimic carcinoma of the breast. Confirmation of the diagnosis tends to be via obtaining culture and sensitivity results of a Salmonella organism and its sensitivity pattern. Treatments that have been given include: Utilization of correct antibiotics based upon the sensitivity pattern of the cultured organism, plus surgical treatment which could be complete aspiration of the pus, incision and drainage of the abscess or in cases where carcinoma of the breast was the provisional diagnosis or the diagnosis is unknown subcutaneous mastectomy and this can be avoided by biopsy of a lesion that mimics a malignant lesion. Follow up of patients is required to ensure the breast lesion has fully resolved. Adoption of the World Health organization guidelines for the prevention of Salmonella infection would help avoid the development of the infection. Urine culture, routine haematology and biochemistry blood tests as well as serology WIDAL tests and stool culture are some of the tests that tend to be undertaken in the investigation of the disease and in order to diagnose the disease a good history would be required including a history of travel from a non-typhoid endemic area to an endemic area
\end{abstract}

\section{Conclusions:}

Salmonella breast abscess / mastitis is an uncommon disease which clinicians should have a high index of suspicion for. A history of previous enteric fever does help in suspecting the disease which at times does need to be distinguished from carcinoma of the breast. The diagnosis is confirmed by obtaining the culture results of the pus from the breast abscess / breast tissue. If all breast lesions are biopsied especially in cases of suspicion of a breast cancer and a past history of abdominal pain, diarrhoea and vomiting the results of the pathology examination of the specimen would negate malignancy and confirm an infection which would help in avoiding mastectomy procedures and enable utilization of antibiotics and drainage/aspiration of abscess to provide adequate treatment.

Key Words: salmonella breast abscess; salmonella mastitis; bacteriology culture; widal tests, stool culture; blood culture; aspiration; incision and drainage; mastectomy; unilateral; bilateral; recurrence; non-lactating; females; males; children; infants

\section{Introduction}

It has been iterated that Staphylococcus aureus is the commonest cause of breast abscess. [1], and additionally, streptococci, gram-negative bacilli, as well as anaerobes could cause breast abscess. [2, 3, 4, 5] Breast abscess that is caused by Salmonella is said to be rare and it tends to occur as a late complication of enteric fever [1,6]. It has been documented that breast abscesses had been reported to ensue Salmonella enterica serotype Typhi and para-typhi infections [1]. Furthermore, the development of breast abscesses that had been related to non-typhoidal Salmonella are very rare. [2,7] Because of global travelling, cases of Salmonella mastitis and breast abscess can be encountered anywhere in the world. 
Nevertheless, because of the rarity of Salmonella infections of the world, it would be envisaged that majority of clinicians globally would not be familiar with the manifestations of the infection. All clinicians need to be familiar with the manifestations, investigation and diagnosis of the commonest type of Salmonella infection in order to have an understanding of the biological behaviour of Salmonella mastitis and Salmonella breast abscess. Additionally, in order that clinicians globally are reminded to have a high-index of suspicion of Salmonella mastitis and Salmonella breast abscess it would be important to provide a review of the literature on the disease entity. The ensuing article on Salmonella mastitis and Salmonella breast abscess is divided into two parts including: (A) Overview which has discussed various aspects of Salmonella infection generally and (B) Miscellaneous narrations and discussions from some case reports, case series, and studies related to Salmonella mastitis and Salmonella breast abscess.

\section{Aims}

To review and update the literature on Salmonella mastitis and Salmonella breast abscess.

\section{Methods}

Various internet data bases were searched including: Google, Google Scholar, Yahoo and PUB MED. The search words that were used included: Salmonella breast abscess, Salmonella mastitis. Twenty nine references were identified which were used to write the review and update of the literature on Salmonella Mastitis and Salmonella breast abscess which has been divided into two parts: (A) Overview which has discussed general aspects of Salmonella infection including Salmonella infections of the bowel and (B) Miscellaneous narrations and discussions related to some case reports, case series, and studies related to Salmonella mastitis and Salmonella breast abscess.

\section{[A] Overview / general statements}

- Salmonella infection is a terminology that is utilized for a disease that is caused by Salmonella species [8].

- It has been iterated that even though overlap does exist in Salmonella infection, the species of Salmonella that causes typhoid fever and non-typhoidal species often tend to be categorized separately [8].

\section{Essential features of Salmonella infection}

- It is well known that Salmonella infection which is also called Salmonellosis is regarded as food borne disease [8].

\section{- Typhoid fever:}

- Generally typhoid fever tends sometimes to be referred to as enteric fever and this disease represents a life threatening disease that is caused by Salmonella typhi and this disease at times tends to be called Salmonella Enterica Serotype Typhi or Salmonella Para-typhi [8]

- It has been iterated that typhoid fever tends to affect between 20 million and 30 million individuals each year and majority of the infections have tended to occur within the developing countries. [8]

- It has been documented that within the industrialized countries of the world, typhoid fever tends to be seen in individuals that travel to other countries. [8]

- Non-typhoid species
- It is known that non-typhoid species of salmonella does usually tend to cause acute, self-limited gastroenteritis. [8]

○ Non-typhoid species of Salmonella has been estimated to account for one million cases, 19,000 hospitalizations and 450 deaths each year within the United States of America. [8]

○ It has also been iterated that tens of millions of cases of non-typhoidal infections do occur globally per year and the disease does cause an estimated 100,000 deaths each year. [8]

○ Non-typhoid infection is stated to spread via ingestion of food or water that has been contaminated and often this tends to occur in sporadic manner; nevertheless, the infection tends to occur via food-borne outbreaks which often tend to be associated with sub-optimal sanitary practices or food preparation. [8]

\section{Terminology}

- It has been iterated that the terminology of Salmonella has been coined after veterinarian Elmer Daniel Salmon [8].

- It has been stated that Salmonella organisms are gram negative rods [8].

- It has been iterated that Salmonella species tend to be serotyped based upon the chemical content of the $\mathrm{O}$ antigens that are found upon the surface as well as the protein content of part of the flagella which is known as $\mathrm{H}$ antigen [8].

- It has been stated that more than 2,500 serotypes of Salmonella had been described; nevertheless, less than 100 serotypes of Salmonella have been known to be associated with Salmonella disease (Salmonellosis) in human beings [8]

- It has been stated that at the moment serotyping of Salmonella is undertaken by means of molecular methods [8].

- It has been documented that Salmonella typhi and Salmonella para-typhi have been associated with typhoid fever [8].

- $\quad$ Some of the documented more common non-typhoidal species of Salmonella include the following: [8]
○ Salmonella. enteritidis
○ Salmonella typhimurium
○ Salmonella muenchen.
○ Salmonella adnatum and Salmonella give.
- Salmonella para-typhi could also cause non- typhoidal Salmonellosis [8]

\section{Epidemiology}

Some of the summations related to the epidemiology of Salmonellosis have been documented as follows: [8]

- $\quad$ Globally more than 20 million cases of typhoid fever tend to be reported every year and worldwide over 20 million cases of typhoid fever have been reported every year and majority of cases of Salmonellosis tend to be reported within the developing countries of the world.

- Nearly 1,800 cases of typhoid fever have been stated to occur within the United States of America each year and these infections mostly tend to be encountered in travellers.

- Between 1.0 million and 1.2 million cases of non-typhoidal Salmonellosis tend to be reported within the United States of 
America each year and these have been reported to be responsible for 19,000 hospitalizations and 450 deaths each year.

- It has been iterated that tens of millions of non-typhoidal Salmonellosis do occur globally and non-typhoidal Salmonellosis do cause more than 100,000 deaths per year.

- Salmonellosis does ensue ingestion of bacteria which usually tend to be from food or water, eggs, milk, meat, as well as poultry.

- Vegetables that had been contaminated with animal faeces tend to be commonly implicated in cases of Salmonellosis.

- Food borne outbreaks of Salmonellosis often tend to be associated with less than sanitary conditions and they tend to be common sources of Salmonellosis. Nevertheless, within the United States of America about $60 \%$ of food borne outbreaks of Salmonellosis tend to occur sporadically.

- It has been iterated that faecal-oral transmission of Salmonellosis could occur and development of Salmonellosis pursuant to contact with infected animals is another source of Salmonellosis.

- It has been documented that Salmonellosis tends to be a common cause of traveller's diarrhoea.

- It has been iterated that all serotypes of Salmonella at least theoretically could cause human Salmonellosis.

- It has been stated that serotypes of Salmonella might or might not be host-specific.

- It has been iterated that when Serotypes that are specific to other species do infect human beings severe disease could emanate.

- It has also been iterated that Salmonella typhi is restricted to human beings.

- It has been iterated that risk factors for the development of Salmonellosis do include the following:[8]

- Lack /absence of access to clean water

- Poor / inadequate sanitation, especially with regard to the handling of food

- Flooding

- The ingestion of plants that have been fertilized with sewage.

○ Urban environments

- Sexual transmission of Salmonellosis has been documented

- It has been iterated that both typhoidal Salmonellosis and nontyphoidal Salmonellosis could occur in health care workers when proper hygiene has not been practiced.

- It has been iterated that travellers to the developing world tend often to be infected with Salmonella para-typhi and hence vaccination against Salmonella typhi tends to be common preceding the travel.

\section{Sites of Salmonella infections}

- With regard to sites of infection of Salmonella, it has been iterated that Salmonella typhi could infect the entire gastrointestinal tract; nevertheless, Salmonella infection tends to be typically found within the terminal ileum, appendix, and ascending colon and that non-typhoidal Salmonella does infect the small and large bowel.

- Salmonella typhi is said to typically spread to the liver, spleen, bone marrow, and lymph nodes [8].

- It has been iterated that extra-intestinal spread of Salmonella tends to be uncommon in non-typhoidal Salmonellosis but this could occur with regard to severe Salmonellosis [8].

- Generally it would be stated that with regard to human beings Salmonella infection does tend to be reported sporadically in almost every organ of the body including: the penis, scrotum, epididymis and testis, urinary bladder as well as the urinary tract organs, the genital tract organs including the ovaries, fallopian tubes, and the uterus, the lungs, the heart, the bones, the brain as well as the breast. Because Salmonella infection only tends to affect the breast on very rare occasions, it would be envisaged that majority of clinicians within the developing countries as well as the developed countries would never have encountered a case before and they would also tend not to be familiar with the manifestations, clinical examination findings and the diagnostic features as well as management of Salmonella infections of these rare sites.

\section{Pathophysiology}

- With regard to the pathophysiology of Salmonellosis, it has been iterated that Salmonella does possess cellular mechanisms that enable bacterial proteins to be transferred to enterocytes as well as $\mathrm{M}$ cells with the subsequent growth within endosomes [8].

- It has also been iterated that subsequent events do include an inflammatory response with neutrophil recruitment as well as mucosal damage [8].

- It has been documented that the host immune response does typically control non-typhoidal infection; nevertheless, the very young individuals, elderly individuals, debilitated as well as immunosuppressed individuals could lack the response that is necessary to control the infection [8].

- It has been iterated that Salmonella typhi do more commonly invade M Cells [8].

- It has also been stated that Salmonella bacteria tend to be phagocytosed by histiocytes within underlying lymphoid tissue [8].

- It has furthermore been stated that the Salmonella bacteria do proliferate and disseminate widely through blood vessels as well as lymphatic channels [8].

\section{Aetiology}

The ensuing summations have been made regarding the aetiology of Salmonellosis: [8]

- $\quad$ Salmonella are known to be gram negative bacilli [8].

- Salmonella typhi tends to be found in human beings [8]

- Non-typhoidal species tend to be found in human beings, domestic animals as well as wild animals [8].

- It has been iterated that generally, Salmonella infection does occur via ingestion of Salmonella contaminated food or water or via the faecal-oral route [8].

- It has also been stated that Non-typhoidal Salmonella tend to be commonly encountered in food as well as companion animals with the inclusion of poultry, cattle, swine, parrots, cats, dogs, as well as turtles [8].

- It has also been documented that eggs, milk, meat, poultry, as well as contaminated vegetables have commonly been implicated in Salmonellosis [8].

- It has been iterated that direct person to person transmission of Salmonella infection from pets could occur and in this setting, the source of the transmission of Salmonellosis might not appear to be ill or may be symptomless [8].

Clinical features of salmonella infections of the bowel (ileum, appendix, and colon)

Symptoms of non-typhoidal Salmonellosis affecting the bowel: 
- The symptoms of non-typhoidal Salmonellosis of the bowel have been stated to generally commence 12 hours to 36 hours pursuant to ingestion of the Salmonella bacteria but this could commence pursuant to from between 6 hours and 72 hours [8].

- Ingestion of very few Salmonella bacteria could cause Salmonellosis [8].

- It has been iterated that typically individuals who are afflicted by non-typhoidal Salmonella infection of the bowel do tend to suffer self-limited illness that tend to manifest with diarrhoea, abdominal pain, fever, nausea, and on rare occasions vomiting which tends to be unpleasant but this tends to be rarely life threatening [8]

- It has been iterated that non-typhoidal infection of the bowel could be life threatening with regard to infants, the elderly, the immunocompromised, and debilitated patients, as a result of dehydration or dissemination of the non-typhoidal bacteria [8].

- It has also been iterated that on very rare occasions, toxic megacolon could complicate the non-typhoidal Salmonellosis of the bowel [8].

- It has been documented that individuals who have achlorhydria or hypochlorhydria from medications, chronic Helicobacter pylori infection or from other causes are at a higher risk for the development of non-typhoidal Salmonella infection [8].

- It has been pointed out that some individuals do develop a reactive arthritis which could last for months as well as lead to chronic arthritis [8].

\section{Symptoms of typhoid enteritis fever}

- It has been iterated that typhoid fever affecting the bowel does cause symptoms shortly after ingestion of the salmonella bacteria [8].

- It has been documented that individuals who are afflicted by Typhoid Salmonella infection of the bowel tend to suffer from severe abdominal pain, bloody diarrhoea, bloating of abdomen, nausea, vomiting, as well as head ache [8].

- With regard to Salmonella typhoid fever infections of the bowel tends to be associated with a brief asymptomatic period that is ensued by bacteraemia with fever as well as flu-like illness [8].

- It has been stated that during the period of fever blood cultures tend almost always to be positive for Salmonella typhi as well as antibiotic treatment could be lifesaving at this stage [8].

- It has additionally been iterated that with progression of the salmonella typhi disease in untreated patients, the development of high fever and abdominal pain does occur which could simulate appendicitis and this could last for about two weeks [8].

- It has been pointed out that when patients survive from the typhoid enteric fever, their symptoms slowly abate [8].

- It has been documented that possible extra-intestinal complications of Salmonella typhi disease of the bowel do include: central nervous disease, endocarditis, myocarditis, pneumonia, cholecystitis, as well as osteomyelitis and that patients who have Sickle cell disease tend to be particularly prone to the development of osteomyelitis [8].

- It has been pointed out that relapses of Salmonella typhoid disease could occur [8].

\section{Diagnosis of Salmonella infection of the bowel.}

The diagnosis of Salmonella infections of the bowel have been summated as follows: [8]

- It has been pointed out that Non-typhoidal Salmonellosis has traditionally been diagnosed based upon the undertaking of stool culture even though many individual patients do not tend to seek any medical attention [8].

- It has been iterated that recently a PCR based assay had been introduced and this in addition to Salmonella species bacteria, also does detect Campylobacter group, Shigella species, Vibrio group, Yersinia enterocolitica, Shiga toxin 1 and 2, Norovirus G1 / GII, Rotavirus A and Aeromonas species [8].

- It has also been stated that serotyping tends to be undertaken to characterize outbreaks of the disease [8].

- It has additionally been iterated that typhoid fever tends to be diagnosed by means of blood culture or stool culture [8].

- It has been pointed out that within the developing countries of the world where laboratory facilities might not be available readily, the disease has tended to be treated empirically [8].

Factors of Prognostication in Cases of Salmonella of the bowel [8]

Non-typhoidal salmonellosis of the bowel [8]

- It has been iterated that non-typhoidal Salmonella infection of the bowel tends almost always to be a self-limited infection, with the exception of infants, the elderly, immunocompromised as well as debilitated patients

- It has also been stated that antibiotic treatment usually tends to be effective in patients who have non-typhoidal disease of the bowel even though the development of resistance of the organism to antibiotics is a growing problem.

Typhoid fever (Salmonella typhoid infection of the bowel [8]

- It has been stated that without treatment of Salmonella typhoid bowel disease, the outcome could be lethal even with regard to healthy patients but the prognosis of the disease tends to be worse in infants, elderly individuals, immunocompromised individuals, as well as debilitated patients.

- It has been pointed out that prompt commencement of antibiotic treatment does greatly improve the outcome of patients, even though the development of antibiotic resistance has tended to be a growing global problem [8].

\section{Treatment of Salmonella infections of the bowel}

The treatment of Salmonella infections of the bowel have been summated as follows: [8]

- Non-typhoidal Salmonella infections of the bowel.

- It has been documented that generally the treatment of non-typhoidal salmonella infections of the bowel does entail supportive care especially hydration of the patients [8].

- It has been iterated that utilization of antibiotics tends to be indicated with regard to severe disease as well as individuals who are vulnerable [8].

- With regard to antibiotics treatment for nontyphoidal Salmonellosis of the bowel, the Center for Disease Control (CDC) has currently recommended utilization of Ceftriaxone as well as ciprofloxacin [8]

- It has been pointed out that antibiotic resistance in cases of non-typhoidal Salmonellosis of the bowel has become a growing problem [8].

- Typhoid fever (Salmonella typhi) infections of the bowel. It has been iterated that with regard to Salmonella typhi infections of the bowel, utilization of antibiotic treatment tends 
to be obligatory and antibiotic treatment should not be delayed in order to obtain confirmation of the laboratory test results [8].

- It has been stated that previously, chloramphenicol, trimethoprimsulfamethoxazole, cephalosporins, as well as first generation fluoroquinolones were administered; nevertheless, the development of resistance to antibiotics has become an issue to appreciate [8].

- With regard to recent treatment of Salmonella typhi infections of the bowel, third generation fluoroquinolones had been recommended; nevertheless, antibiotic resistance has also been reported with these recommended treatments [8].

- It has been iterated that the undertaking of surgical operation could be necessitated for perforation of the bowel or for gall bladder disease [8].

- It has additionally been documented that an effective vaccine for Salmonella typhi does exist [8].

- The World Health Organisation (WHO) recommendations for the Public as well as travellers do include the ensuing: [8]

- The public as well as travellers should ensure their food is properly cooked as well as hot when the food is served.

- The public as well as travellers should avoid drinking/eating raw milk as well as products that are made from raw milk.

- The public and travellers should avoid ice unless the ice is made from safe water.

- The public and travellers should utilize disinfecting tablets for water unless the purity of the water is certain.

- The public as well as travellers should thoroughly wash their hands with soap and water pursuant to contact with pets or farm animals and following their use of the toilet.

- The public and travellers should wash their fruits as well as vegetables thoroughly and if it is possible to peel fruits and vegetables unless they are certain that optimal sanitary practices have taken place.

- Guiding Recommendations for food handlers and producers do include the following: [8]

○ Food handlers as well as food producers should maintain a clean workspace

- Food handlers as well as food producers should separate their raw and cooked food.

- Food handlers and food producers should cook their food thoroughly

- Food handlers and food producers should keep their food at safe temperatures

- Food handlers and food producers should utilize safe water as well as raw materials.

- Food handlers and food producers should practice good personal hygiene.

- Food handlers and food producers should not work if they develop the ensuing: fever, diarrhoea, vomiting, or infected skin lesions.

- Food handlers and food producers should protect their fields from contamination by animals.
Macroscopic gross examination features of the bowel in Salmonella infections of the bowel. [8]

- It has been iterated that the undertaking of colonoscopy in individuals who have mild non-typhoidal Salmonella infection of the bowel does demonstrate non-specific findings that tend to include oedema as well as petechial haemorrhage which does tend to be more associated with friability as well as ulceration in more severe salmonella diseases.

- It has been documented that typhoid fever does cause marked enlargement of Peyer patches as well as the lymphoid tissue within the appendix as well as the ascending colon which tend to lead to elevation of the mucosa along the axis of the ileum.

- Typhoid perforation of the bowel could occur and this would be visualised and may be responsible for visible evidence of peritonitis.

- Macroscopic examination could reveal presence of small, grey soft nodules that are referred to as typhoid nodules within the liver.

- Macroscopy examination also does demonstrate splenomegaly as well as lymph node enlargements.

Microscopy histopathology examination features of Salmonella infections of the bowel: [8]

- It has been iterated that non-typhoidal Salmonella infections tend to be rarely biopsied and that the pathology examination findings in cases of non-typhoidal infections of the bowel tend to be non-specific self-limited colitis.

- It has been documented that with regard to cases of nontyphoidal Salmonellosis of the bowel, microscopy pathology examination of the part of the bowel affected does show that the crypt architecture is maintained and there tends to be increased amount of mixed inflammatory cells within the lamina propria in a patchy distribution that is associated with foci of cryptitis as well as presence of possible crypt abscesses.

- With regard to long-standing cases of non-typhoidal infections of the bowel, microscopy pathology examination could show evidence of architectural distortion which raise the spectre of idiopathic inflammatory bowel disease and this is said to be also true for Salmonella typhimurium infection.

- It has been iterated that knowledge of positive bacteriology culture results of Salmonella is necessary in order to confirm a definitive specific diagnosis of Salmonella infection.

- It has been documented that microscopy examination of the bowel that has been affected by Salmonella typhi does show neutrophils, histiocytes, with cytoplasmic bacteria, nuclear debris, as well as haemorrhage within the lamina propria that is associated with presence of a lympho-plasmatic infiltrate.

- In Salmonella typhi infections microscopy examination does show that the spleen and lymph nodes do demonstrate sinus histiocytosis which does distort the normal architecture of the spleen and the lymph nodes.

- Microscopy examination of typhoid liver nodules does demonstrate aggregates of histiocytes with necrotic debris.

- It has been iterated that typhoid nodules could also be found within bone marrow as well as within lymph nodes.

Differential diagnosis of Salmonella infections of the bowel.

- It has been stated that the differential diagnosis of nontyphoidal Salmonella infection does include other enteric pathogens which cause acute self-limited colitis and some of these include Shigella and Campylobacter [8] 
- It has also been stated that long-standing cases of non-typhoidal Salmonella infections of the bowel could simulate idiopathic inflammatory bowel disease [8].

Definition / General Comments Related to Salmonella of the breast

Salmonella of the breast is very uncommon.

- The incidence of mastitis / breast abscess in patients who have typhoid has been stated to be $0.3 \%$ in 1930 by Close and Sebeng and $0.5 \%$ in 1937 by Peziski in a study of 1,196 cases of typhoid fever over a period of 2 years. [9]

- With regard to females, the incidence of Salmonella infection of the mammary gland was $0.90 \%$ [9] [10] [11] [12] Razeq and associates [13] and Edelstein [2] had isolated Salmonella Landwasser and Serogroup B in breast abscess respectively.

- Salmonella infection of the breast could be:

$\begin{array}{ll}\circ & \text { Acute } \\ \circ & \text { Chronic } \\ \circ & \text { Unilateral } \\ \circ & \text { Bilateral }\end{array}$

- Salmonella mastitis / abscess can be seen in:

○ The female

- The male on rarer occasions

- Salmonella mastitis / abscess may be found in the following:

- A healthy individual who has no history of any significant co-morbidity

- An individual who is known to have diabetes mellitus

- Immunocompromised individual

$\circ$ An individual who has had a history of salmonella infection of the bowel recently or many months earlier that usually had not been treated. There may not be a definite history of previous diarrhoea or salmonella mastitis / abscess at the time of presentation of the disease but when the diagnosis of Salmonella infection of the breast has been established based upon microbiology culture of Salmonella organism from the aspirate of the breast abscess or breast specimen, a review of the past medical history of the patients tends to reveal a past history of diarrhoea / diagnosis of previous Salmonella infection that had not been treated.

- Cases of salmonella infection of the breast could be found in some individuals who have rheumatoid arthritis or fibroadenoma of the breast.

- With regard to ladies who have Salmonella mastitis / abscess these usually tend to occur in non-lactating women in comparison with staphylococcus mastitis / breast abscess which tends to occur in lactating women. Salmonella infection of the breast can also affect children and infants.

- Salmonella mastitis / breast abscess could be due to Salmonella typhi or salmonella para-typhi infection.

\section{Presentation of cases of Salmonella of the breast}

The presentation of salmonella infection of the breast in the form of mastitis or breast abscess does vary from one individual to another and the presentation tends to be non-specific and does simulate the presentation of various breast conditions but generally Salmonella infection of the present could manifest as follows:
- Lump / Mass - The finding of a lump/mass within the breast of a few days duration or the breast lump could be present over a long-period of time.

- Discomfort / Pain in breast - The lump / mass in the breast may be associated with mild discomfort or pain.

- No nipple discharge - There tends not to be any discharge associated with the lump/mass

- Nipple discharge - Few of the reported cases have been associated with discharge from the nipple but majority have not been associated with discharge.

- $\quad$ Sex of patient - majority of patients who have been reported as having Salmonella mastitis / Salmonella breast abscess have tended to be female but salmonella infection / abscess of the breast has also been reported in males.

- Age - Salmonella infection of the breast can affect individuals including children and adult.

- Fever - some cases of Salmonella of the breast do not tend to be associated with fever but in some cases there may be an antecedent history of low-grade or high fever that may be antecedent or may exist contemporaneously with pain / lump / mass within the breast.

- Vomiting - In some cases of Salmonella mastitis or Salmonella breast abscess there could be an antecedent history of gastroenteritis type of symptoms that include vomiting and on a number of occasions the patient who has had vomiting may not report a previous history of vomiting and often a history of previous vomiting may be obtained after the diagnosis of salmonella abscess / mastitis has been made in a retrospective history taking.

- Diarrhoea - A number cases tend to be associated with a previous episode of loose stool or diarrhoea which may have settled and the individual patient may have forgotten about the diarrhoea; nevertheless, in other patients there could be contemporaneous episodes of diarrhoea.

- Abdominal pain / discomfort - Some individuals may have a past history of abdominal pain / discomfort and occasionally there could be concurrent abdominal pain / discomfort that may tend to be associated with fever, vomiting / diarrhoea but is often not the case of some patients who would not report abdominal pain.

Clinical examination findings in cases of Salmonella infections of the breast

Some of the findings on clinical examination in cases of Salmonella breast abscess include the following:

- $\quad$ Lump / mass within one breast or both breasts which may or may not be tender.

- Nipple retraction is not common but some individuals may have retraction of nipple

- Majority of cases tend not to be associated with nipple discharge but in some rare cases, there may be nipple discharge of the abscess is in the areolar region.

- Inflammation of the breast - With regard to majority of cases of Salmonella breast abscess there tends not to be any superficial inflammation of the breast but in cases of Salmonella mastitis, there may be evidence of inflammation.

- Hard lump - In some cases of Salmonella breast abscess the breast lump/mass may feel firm/hard and this could simulate carcinoma of the breast / tuberculosis of the breast. 
- Soft mass within the breast - This would tend to be fluctuant giving the impression of an abscess and following aspiration of the abscess or drainage of the abscess bacteriology culture of Salmonella would be obtained to establish the diagnosis.

- Axillary lymph node - Majority of cases of Salmonella mastitis / breast abscess would not be associated with lymph node enlargement in the axilla but some cases have been reported with the finding of a palpable lymph node with a breast mass and this could mimic carcinoma of the breast or tuberculosis of the breast.

- Fever-majority of reported cases tend not to be associated with fever but cases of Salmonella breast abscess / mastitis which have quickly developed contemporaneously with bacteraemia associated with enteric fever may be associated with the finding of pyrexia and in such cases a history of abdominal pain, vomiting and diarrhoea may be obtained.

- Dehydration - Majority of cases of Salmonella breast abscess / mastitis would tend not to be associated with dehydration but occasionally when the abscess / mastitis develops in association with enteric fever bacteraemia there could be evidence of dehydration.

- Weight loss - Occasionally there could be evidence of weight loss in comparison with the previous weight of the patients. .

Laboratory investigations in cases of Salmonella abscess of the breast / mastitis

\section{Microbiology tests}

Urine

Urinalysis, urine microscopy and culture are tests that tend to be undertaken as part of the general assessment of all individuals who have Salmonella mastitis or Salmonella breast abscess but the results would tend to be normal and if there is evidence of urinary tract infection this would be treated with appropriate antibiotics to help improve the general condition of the patients but the results would not be diagnostic of Salmonella abscess / of the breast or mastitis.

\section{Stool}

On the whole the results of stool culture in cases of Salmonella mastitis / breast abscess would tend to be negative for Salmonella infection but there could be a past history of a positive stool test result for Salmonella which the patient may have forgotten about but on rare occasions of the manifestation of the Salmonella breast abscess / mastitis being synchronous with an episode of Salmonella gastro-enteritis Salmonella could be isolated from the stool specimen but this is very rare.

\section{Blood culture}

Quite often during an episode of Salmonella breast abscess, blood cultures tend to be negative but in cases of a previously investigated Salmonella gastro-enteritis that was associated with bacteraemia, blood culture that is undertaken during the previous bacteraemia phase of the disease blood culture could yield a growth of Salmonella but this may not be remembered during the manifestation of Salmonella abscess of the breast and usually a retrospective review of the previous medical history of the patient could lead to the finding of positive Salmonella culture in the results of the patient's past investigations.

\section{Haematology blood test}

In cases of Salmonella abscess of the breast / salmonella mastitis, the results of full blood count could be normal and on other occasions there is the possibility that the white blood cell count level could be raised but the results would not be diagnostic of Salmonella abscess / mastitis. Nevertheless, the results would tend to form part of the general assessment of all the patients.

\section{Biochemistry blood tests}

Routine biochemistry blood tests in cases of Salmonella breast abscess / mastitis tend to be normal but if there are diseases that affect the renal function or liver function test results these would be reflected in the results but the results themselves would not be diagnostic of Salmonella breast abscess or mastitis.

\section{Widal test}

Widal test is a serological test for enteric fever. Named after the inventor, Georges -Fernand Widal test is an indirect agglutination test for enteric fever or undulant fever and this test tends to be positive in cases of enteric fever. This test tends to form part of the assessment of patients who are suspected to have Salmonella breast abscess / mastitis but in a number of cases culture of the breast abscess would grow the Salmonella species but the Widal test which would be expected to be positive tends to yield a negative test result. This means that the finding of a negative Widal test does not mean that the breast abscess is not Salmonella abscess therefore it is important to send all specimens of the breast abscess for culture and sensitivity to confirm the result.

\section{Radiology imaging}

\section{Chest X-ray}

Chest X-ray tends to be undertaken at times when the lesion within the breast mimics a malignant lesion of the breast and this could form part of an initial screening in some parts of the world to ascertain if the individual has got metastatic lesion within the lungs but this has been superseded by CT scan of the thorax. However, in cases of Salmonella infection of the breast the chest $\mathrm{x}$-ray would tend to be normal.

\section{Mammography}

Mammography has been undertaken in some cases of Salmonella breast abscess taking into consideration the suspicion of the lump/mass being a possible breast cancer but the features of the mammography would tend to demonstrate features that tend not to be suspicious of breast cancer.

Ultrasound scan

Ultrasound scan of the breast in cases of Salmonella abscess of the breast / mastitis tend to demonstrate the lesion within the breast and its echogenicity and most often does demonstrate the fluid nature of the breast lesion and based upon the fluid nature of the breast lump, aspiration / incision and drainage of the abscess can be undertaken as treatment as well as to submit the push for culture and sensitivity which would confirm the diagnosis.

\section{Computed Tomography (CT) scan}

Computed tomography scan of the breast / thorax would define the nature of the breast lesion which would tend to confirm non-contrast enhancing lesion as well as illustrate features that generally tend not to be suspicious of a malignant lesion within the breast. In cases that are suspicious for carcinoma of the breast based upon clinical examination CT scan of the thorax would tend to show normal findings within the lungs and thorax.

\section{Magnetic Resonance Imaging (MRI Scan}

Magnetic Resonance Imaging (MRI) scan of the breast / thorax would define the nature of the breast lesion which would tend to confirm noncontrast enhancing lesion as well as illustrate features that generally tend not to be suspicious of a malignant lesion within the breast. In cases that are suspicious for carcinoma of the breast based upon clinical examination 
MRI scan of the thorax would tend to show normal findings within the lungs and thorax.

Diagnosis

Diagnosis of Salmonella breast abscess / mastitis tends to be confirmed based upon bacteriology culture of aspiration specimens of the breast abscess and at times following pathology examination and microbiology culture of specimens of the breast lesion(s) that had been obtained by biopsy or mastectomy.

\section{Treatment}

Treatment of patients who have Salmonella breast abscess / mastitis have included a combination of:

[1] The undertaking of Surgical treatment including:(a) complete aspiration of the breast which in a number of cases has been under ultrasound-scan guidance but in areas of the world where ultrasound scan facilities are not available by palpation to find the fluctuant lesion, (b) incision and drainage of the abscess (c) mastectomy (sub-cutaneous at times) when the lesion has been provisionally diagnosed as carcinoma of the breast and it is only after the results of the histopathology examination and bacteriology culture is obtained that the diagnosis of an abscess is made.

[2] Antibiotics - Utilization of correct antibiotic treatment based upon the sensitivity pattern of the cultured organism

\section{Outcome}

- Following utilization of aspiration or incision and drainage of the Salmonella breast abscess in combination with correct antibiotic treatment based upon the sensitivity pattern of the disease majority of the cases completely resolve.

- Nevertheless, occasional cases of recurrence of the breast abscess have been reported and factors for the development of recurrence of the abscess include (a) incomplete drainage of the abscess, (b) utilization of an antibiotic which the Salmonella organism is not sensitive to, (c) non-compliance to completing a full course of the antibiotic.

- In some few reported cases when the breast abscess had been provisionally diagnosed as adenocarcinoma of the breast and mastectomy had been undertaken including sub-cutaneous mastectomy even though after the diagnosis is established and antibiotic treatment is given that leads to complete resolution of the abscess, it would be said that the treatments would then have resulted in overtreatment and cosmetic problems for some patients.

\section{Differential diagnoses of Salmonella abscess of the breast / mastitis}

Some of the differential diagnoses of Salmonella breast abscess include: Carcinoma of the breast, chronic inflammatory lesions of the breast / tuberculosis of the breast.

\section{[B] Miscellaneous narrations from some case reports, case} series and studies undertaken in relation to Salmonella mastitis and Salmonella breast abscess

Al-Ishak et al [14]. reported the case of Salmonella enterica subsp enterica (1) serovar Enteritidis breast abscess in a 48-year-old woman who is a citizen of United Kingdom and who had travelled abroad. She was treated successfully with utilization of surgery and ciprofloxacin antibiotic treatment. Al-Ishak et al [14]. Advised that unusual causative organisms should be borne in mind with regard to patients who had recently travelled abroad as well as pus should be sent for culture and sensitivity. They iterated that Mastitis that is caused by Salmonella spp. could manifest with a severely indurated area which could take a few weeks to resolve. They additionally stated that complete assessment biopsy of the indurated area as well as imaging of the breast is mandatory in order to exclude malignancy of the breast.

Agrawal et al. [15] reported 2 cases of breast abscesses that were caused by salmonella enterica serotype para-typhi as follows:

\section{Case 1}

A 27-year-old lady presented with painful lump within her left breast of 2 months duration. She reported that the lump started to grow bigger in size gradually over the preceding 2 months. She stated that the lump was in the first instance firm as well as painless; nevertheless, the lump did gradually grow in size and it became painful. She was not pregnant as well as she was not lactating at the time of her presentation. She was asymptomatic otherwise and she did not have any other co-morbidities. Her general and systematic examinations were within normal range. Clinical examination revealed that her left breast was tender and swollen. A mobile, soft, fluctuant mass that measured about $5 \mathrm{~cm} \times 6 \mathrm{~cm}$ that was located within the lower quadrant of the left breast was found by palpation of her breast. The overlying skin of her left breast was noted to be warm as well as erythematous. No spontaneous discharge was observed from the abscess and there was no evidence of lymphadenopathy. The nipple was not retracted as well as there was no discharge from the nipple. Her axillary nodes were not palpable. She had ultrasound scan (USG) of her left breast which showed a heterogeneously hypo-echoic deep-seated irregular collection that measured about $4.9 \mathrm{~cm}$ x $3.5 \mathrm{~cm}$. Based upon the features of her breast lesion, a diagnosis of breast abscess was made. The breast abscess was drained under ultrasound-scan-guidance and she was empirically commenced on amoxicillin-clavulanic acid 628 milligrams 8 hourly. The results of her laboratory tests showed a total leucocyte count of 12,500 / cubic millimetres, with $70 \%$ polymorphonuclear leucocytes. The aspirated pus was sent for microbiology examination. Ziehl-Neelsen staining of the pus did not demonstrate any evidence of acid fast bacilli (AFB) and GeneXpert test was negative for Mycobacterium tuberculosis. Gram staining of the pus did reveal numerous polymorphonuclear leucocytes as well as gram-negative bacilli. The culture of the pus grew cream-coloured non-haemolytic colonieson 5\% sheep blood agar and non-lactose-fermenting colonies on MacConkey agar which were identified to be Salmonella enterica para-typhi A by means of matrixassisted laser desorption ionization-time of flight mass spectrometry with utilization of the bioMérieux VITEK MS system (IVD data base version 2.0) (USA). The bacteria was found upon sensitivity testing to be sensitive to ciprofloxacin, trimethoprim-sulfamethoxazole, erythromycin, azithromycin, chloramphenicol, ceftazidime, ceftriazone, ampicillin as well as Nalidixic acid. As a result of the diagnosis, she was recalled and her past medical history was reviewed which revealed that she had gastroenteritis three months earlier which did resolve without any antibiotic treatment. Her stool, urine, and blood samples were obtained and submitted for culture but the results were negative for Salmonella para-typhi a spp. She had a Widal test which was found to be negative. She was subsequently treated with Azithromycin $1000 \mathrm{mg}$ tablet once daily for five days. She responded well to treatment. Pursuant to ultrasound scan-guided aspiration of the pus and antibiotic treatment, she was cured and the abscess did not recur up to her one-year follow-up assessment.

\section{Case 2}

A 29-year-old lady did present with a lump within her left breast which she had developed over the preceding 20 days. She was not pregnant as well as she was not lactating at the time of her manifestation. Her breast examination revealed a lump that measured $4.5 \mathrm{~cm} \times 3.5 \mathrm{~cm}$ within her left breast at 2 o'clock position that was free and not attached to the skin of her left breast as well as the underlying muscle. The skin overlying the 
breast lump appeared normal with no evidence of inflammation as well as no tenderness. The nipple was not retracted and there was no discharge from her nipple. There were no palpable axillary lymph nodes and the rest of her general examination as well as systemic examination were normal. A provisional diagnosis of breast lump that was suspicious for malignancy was made. She had routine blood tests and the results were normal. She had chest x-ray, ultrasound scan of both breasts, as well as core needle biopsy of both of her left breast lump. Ultrasound scan of her left breast showed heterogeneously hypo-echoic mass lesion that measured about $3.4 \mathrm{~cm} \times 2.4 \mathrm{~cm}$ within her left breast at about 2 o'clock position. Pathology examination of the core needle biopsy of the left breast lump showed plenty of neutrophils as well as inflammatory cells that contained necrotic background suggestive of breast abscess. The lump was excised by means of radical duct excision. She was empirically treated by means of erythromycin $500 \mathrm{mg}$ orally 6 hourly as well as metronidazole $400 \mathrm{mg}$ twice daily for two weeks. The pus that was drained was sent for microbiology pathology examination. Gram staining of the drained pus demonstrated gram-negative bacilli. The culture on blood agar grew grey-white opaque, non-haemolytic colonies as well as non-lactose fermenting colonies on Mackonkey's agar. The non-lactose fermenting gram negative bacillus was found to be Salmonella para-typhi A based upon mass spectrometry testing which was sensitive to the following antibiotics: Ciprofloxacin, trimethoprim-sulfamethoxazole, erythromycin, azithromycin, chloramphenicol, ceftazidime, and ceftriaxone, ampicillin, as well as nalidixic acid. She had blood culture and Widal test were undertaken and the results were negative. She received oral ciprofloxacin $500 \mathrm{mg}$ twice per day for 14 days. She had ultrasound scan of her breast two weeks pursuant to her antibiotic treatment which did not demonstrate any significant change in the heterogeneous hypo-echoic lesion. She underwent left breast lumpectomy with left radical milk duct excision. Some pus-like material that was obtained during the surgical procedure upon culture and sensitivity was found to have grown Salmonella para-typhi A that had similar anti-microbial sensitivity pattern. She received intravenous ceftriaxone 2 grams 12 hourly for seven days. Her follow-up assessment demonstrated complete resolution of the abscess.

Some of the lessons learnt from these 2 cases include the following:

- Salmonella infections of the breast could affect non-lactating individuals who may present with lump within the breast that may gradually grow bigger and which may at times be associated with mild tenderness and no acute pain.

- Generally patients who have mastitis of the breast or abscess of the breast due to Salmonella infection would tend to be generally well.

- Mammography and ultrasound scan of the breast tend to define the lesion within the breast but the findings tend to be nonspecific but the localization of the disease would allow for aspiration / drainage / complete excision of the lesion.

- Diagnosis of the disease tends to be confirmed based upon bacteriology culture of Salmonella.

- Salmonella mastitis / breast abscess does simulate staphylococcus abscess / infection of the breast, chronic inflammation / abscess of the breast including tuberculosis, as well as carcinoma of the breast.

- Treatment of the disease could entail (a) antibiotic treatment plus aspiration of the pus or (b) antibiotic treatment plus aspiration of the pus plus surgical excision of the lesion.

- Widal test, tuberculosis tests as well as routine haematology and biochemical tests are required for the general assessments of all patients who have salmonella infection of the breast.

- Because carcinoma of the breast is one of the differential diagnosis of Salmonella infection of the breast, chest X-ray, CT
Scan of thorax, or MRI scan of the thorax could be undertaken in the initial assessment of patients to see if they have lung metastasis and the results would show normal findings as well as no evidence of pulmonary tuberculosis.

Baran et al [1] reported a 43-year-old woman who presented with a lump, fever and mild pain in her breast. She was not pregnant or lactating at the time of her presentation. She did have a history of rheumatoid arthritis for 5 years and she was undergoing immunotherapy. She had ultrasound scan of her breast which demonstrated breast abscess. The abscess was drained and submitted for culture and sensitivity and Salmonella enterica serotype typhimurium was identified. The organism was sensitive to all of the antibiotics that were tested. The patient was cured after surgical debridement and antibiotic therapy. The abscess did not recur pursuant to treatment. Baran et al [1]. Presented the case to draw attention to nontyphoidal Salmonella as an uncommon cause of breast abscess and they recommended the submission of specimens for bacteriological examination as well as treatment especially in cases of individuals who have underlying immunosuppressive diseases. Some of the lessons learnt from this summation include:

- The fact that some cases of salmonella infection/abscess of the breast could manifest with fever and a painful lump within the breast.

- The disease could simulate other causes of breast abscess including staphylococcus abscess.

- Bacteriology culture of a specimen of the breast abscess is a definite way to confirm the diagnosis of Salmonella infection / abscess of the breast.

Viswanathan et al [16]. reported a 42-year-old married non-lactating lady who had noticed a swelling in her right breast about 15 days preceding her presentation in the hospital. She stated that she had high-grade fever and malaise preceding the swelling within her right breast. She also stated that she had noticed gradual increase in the size of her breast swelling that was associated with mild pain and no evidence of discharge from her nipple. She did not have any past history of breast disease. She did not have any history of loose motions, constipation or urinary abnormality and she had not received any TAB vaccination. She did not have any history of tuberculosis or contact with anyone who had tuberculosis. She did not have any menstrual irregularities as well as she was nulliparous, non-lactating at the time of her manifestation. She did not have any family history of any breast disease. She was found to be non-febrile, and her general and systematic examinations were normal. Her breast examination showed a single, firm, swelling that measured $5 \mathrm{~cm}$ x $6 \mathrm{~cm}$ x $5 \mathrm{~cm}$ within the upper outer quadrant of her right breast that was minimally tender, mobile, and non-adherent to the skin. The skin over the lump was normal but mildly dilated veins were visualised. The areola was normal and there was no discharge noticed from the nipple. Central group of her right axillary lymph nodes were palpable and her left breast did contain a small swelling that measured approximately $2 \mathrm{~cm} \mathrm{x} 1 \mathrm{~cm}$. Her left axilla was normal. No abnormality was found in her respiratory, alimentary, nervous as well as musculoskeletal systems. The differential diagnoses that were considered included chronic breast abscess and primary carcinoma of the breast. Diagnostic aspiration of the breast lesion was undertaken which was sent for culture and sensitivity. She was commenced on Amoxycillin and Clavulinic acid combination. Her haemoglobin level was 11.2 grams\%, white blood cell count 7,500 (59\% neutrophils). Culture and sensitivity of her aspirated pus from the breast grew a pure growth of Salmonella typhi which was sensitive to Ciprofloxacin, Ofloxacin, Norfloxacin, Ceftizomine, Cefuroxime, Ceftazidime, Cefaclor, Amikacin, Gentamycin, Netilmycin, Tobromycin, Piperacillin, Amoxycillin, Amoxycillin + Clavulinic acid, Ampicillin + Sulbactam, Cotrimoxazole, and Chloramphenicol. She had Widal test which revealed titres of Salmonella typhi H 1: 120, 0 1: 120. The titres of 
Salmonella para-typhi A and para-typhi B were nil. She had blood culture which did not yield any growth. Her urine and stool culture did not grow any pathogenic organism. The results of her biochemistry blood tests, Chest X-ray, and ECG were all normal. She had mammography which showed 2 cysts within her right breast that measured $2.4 \mathrm{~cm} \times 1.9 \mathrm{~cm}$ each and a cyst in her left breast that measured $3.0 \mathrm{~cm} \mathrm{x} 1.1 \mathrm{~cm}$. She had ultrasound scan of her breasts which showed $2.1 \mathrm{~cm} \mathrm{x} 1 \mathrm{~cm}$ and $3.5 \mathrm{~cm} \mathrm{x}$ $1.2 \mathrm{~cm}$ cysts in her right breast and left breast respectively. She also had ultrasound of her abdomen which did not reveal any abnormality. She was treated conservatively by means of Ciprofloxacin $500 \mathrm{mg}$ twice daily for 14 days and the size of cysts decreased and did not have any fever or systemic complaints. Repeat aspirations from the cysts were sent for culture which did not grow any organism. Upon subsequent examination, no further decrease in the size of the cyst was noted. The cyst was excised surgically. There was no growth in the culture of the specimen. Histopathology examination of the wall of the abscess demonstrated chronic inflammation.

Seah et al [17]. Reported a 7-week-old Chinese female who was admitted following her development of fever of 1 day duration that was associated with 2 episodes of vomiting as well as reduced-feeding. She was found to have a furuncle of her right breast. She did not have any history of travelling or any relevant history of contact. No members of her family had diarrhoea. She did not consume any raw food or unpasteurized milk but was fed with expressed breast milk. There was a report of insufficient bottle hygiene in that her feeding bottle was rinsed only with hot water between expressed milk feeds. The results of her C-reactive protein (11.6 $\mathrm{mg} / \mathrm{DL}$ ) as well as her white blood cell count of $16.48 \times 10$ to the power 9 per litre were slightly raised. She had ultrasound scan of the breast which demonstrated a $0.5 \mathrm{~cm}$ x $0.5 \mathrm{~cm}$ abscess within her retro-areolar region. An aerobic blood culture was undertaken prior to the commencement of parenteral antibiotic treatment with ampicillin and cloxacillin which demonstrated a growth of Group C salmonella that was sensitive to ampicillin, ceftriaxone, and trimethoprim-sulfamethoxazole. Incision and drainage of the right breast abscess was undertaken on the $2^{\text {nd }}$ day of her admission and culture of the specimen grew Group C Salmonella with the same antibiotic sensitivity pattern. Both isolates of the cultured organisms were typed to be Salmonella Mbandaka by Singapore National Public Health Laboratory. Her urine culture did not grow any organism. All her investigations were normal. Her pyrexia lysed on the $2^{\text {nd }}$ day of her admission and a repeat blood culture was undertaken on the third day of her admission which did not grow any organism. Her antibiotic treatment was changed to ceftriaxone on the $4^{\text {th }}$ day for clearance of possible biliary carriage of non-typhoidal Salmonella and also in order to ease her intramuscular administration of antibiotics due to her poor venous access. She initially developed mild diarrhoea of 5 loose stools on the $6^{\text {th }}$ day of her admission. She was well and was discharged on the $9^{\text {th }}$ day of her admission and completed 2 weeks of ceftriaxone which was ensued by 1 week treatment of oral trimethoprim sulfamethoxazole. Lessons learnt from this summation include:

- Children including infants can develop salmonella infection / abscess of the breast and this could manifest as an acute abscess that can simulate Staphylococcus infection / abscess of the breast.

- Salmonella abscess of the breast can successfully be treated by means of antibiotic treatment and incision and drainage of the abscess.

- In cases of salmonella abscess of the breast, culture of aspirate or the drained abscess would grow Salmonella organism and in some acute cases, blood culture that is undertaken before commencement of antibiotic treatment could culture the Salmonella organism.
Singh et al [9]. reported a case of bilateral breast abscesses that were caused by salmonella enterica serotype Typhi in a non-lactating 29-year old patient who had manifested with a lump in both breasts of 7 days duration. She also had intermittent discharge of pus-like substance from her nipples that was milky with regard to consistency. She also had fever of 1.5 months duration which was high-grade with a peak of 39.0 degrees centigrade which was brought down to normal range with antipyretics. She also did have episodes of loose motions over the preceding one month. She was commenced on co-amoxiclav (amoxycillin and clavulanic acid) $625 \mathrm{mg}$ orally twice daily for 7 days but her symptom of pyrexia did not respond to the treatment. Her blood culture which was taken at home grew Salmonella Typhi which was resistant to amoxycillin but she did not attend the hospital to obtain a new medication. She was not pregnant and she was not lactating. Her clinical examination demonstrated a centrally located lump in her left breast that measured about $8 \mathrm{~cm} \times 8 \mathrm{~cm}$ as well as another lump in her right breast that measured about $3 \mathrm{~cm} \times 3 \mathrm{~cm}$. Within her left axilla a palpable lump that measured $2 \mathrm{~cm}$ was found. Pus was drained from both breast lumps which on culture grew non-lactose fermenting colonies. The organism was identified as Salmonella Typhi which was sensitive to chloramphenicol, cefotaxime, ciprofloxacin, as well as cotrimoxazole, but which was also resistant to ampicillin and nalidixic acid. She also had a report of Salmonella typhi positive culture 2 weeks preceding her manifestation that was sensitive to the same antibiotics. The result of her Widal tests was normal. The results of her routine haematology and biochemistry blood tests were within normal range with the exception of serum glutamic oxaloacetic transaminase (SGOT) and serum glutamic pyruvic transaminase (SGPT) which were raised with values of 96.59 and 113.78 $\mathrm{U} / \mathrm{L}$, respectively. She was commenced on oral ciprofloxacin $500 \mathrm{mg}$ orally twice per day for 2 weeks to which she responded favourably with regression of the lesion. The results of her stool and urine culture were negative. Singh et al [9]. Made the ensuing conclusions:

- $\quad$ Any breast abscess that is found in a non-lactating woman with a history of typhoid fever within the recent past as well as no other predisposing factors should be evaluated whilst keeping in mind the possibility of Salmonella breast abscess.

- Also a combination of medical and surgical management does help in such cases when they are supported by a microbiological culture and sensitivity report.

Some of the lessons that need to be learnt from this summation include:

- Some cases of salmonella breast abscesses may be associated with discharges of pus-like material from the nipple.

- $\quad$ Some cases of Salmonella breast abscesses may be associated with normal full blood count results.

- $\quad$ Some cases of Salmonella breast abscesses may be associated with normal Widal test results.

- $\quad$ Some cases of Salmonella breast abscesses may be bilateral abscesses and whilst a number of cases of Salmonella breast abscesses may not be associated with enlarged axillary lymph nodes, others may be associated with an enlarged axillary node.

Murugesan et al [18]. reported a 60-year-old diabetic woman who was admitted because of fever over a period of 10 days which was ensued by pain and lump within her right breast for the preceding 2 days. She was afebrile and was found to have a lump that measured $7 \mathrm{~cm}$ by $5 \mathrm{~cm}$ within the upper outer quadrant of her right breast that was firm with regard to consistency and with no signs of inflammation. She had ultrasound scan of the breast which showed a right breast abscess which was subsequently treated by incision and drainage and the pus was submitted for culture and sensitivity. Salmonella typhi was cultured from the pus (see figure 1) that was sensitive to ampicillin-sulbactum as well as amikacin. His Widal test 
and blood culture were negative. He did completely recover with drainage

of the abscess and antibiotic treatment.

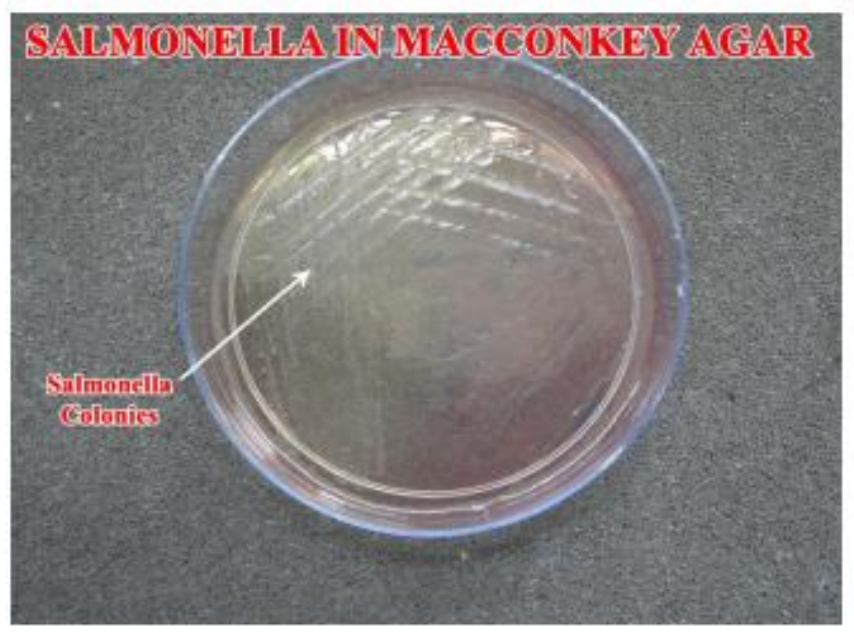

Figure 1: Shows growth of Salmonella colonies in Mac-conkey agar plate. Reproduced from: [18] Murugesan N. Alagar Samy R, Viswanathan M S, Anbazhagan A. A Rare Case of Breast Abscess Due To Salmonella Typhi. Int J Cur Res Rev 2016 Mar; 8(5): 10 - 12. http://ijcrr.com/uploads/317 pdf.pdf

Some of the lessons that had been learnt from this case report include:

- Patients who have Salmonella abscess of the breast may be afebrile.

- Some cases of Salmonella abscess of the breast may not have any signs of inflammation of the skin over the breast abscess

Hall et al [19]. reported the first case of traveller's diarrhoea which had been ensued by breast implant infection. They reported an otherwise healthy 34-year-old woman, who had undergone breast augmentation with a left cremasteric lift (see figure 2). Five months pursuant to her breast operation, she was having a vacation in Cancun, Mexico, when she did develop abdominal pain as well as diarrhoea which had progressed to include fevers as well as chills. Her symptoms did persist until she returned to the United States of America. She was assessed by her primary care physician on the $4^{\text {th }}$ day of her illness. She had computed tomography scan of her abdomen which did not demonstrate any abnormality. The results of her routine biochemistry blood tests did demonstrate raised levels of transaminase. Her symptoms did resolve soon. Fourteen days pursuant to the resolution of her symptoms, she did develop pain within her right breast (see figure 3). Upon examination, her breast was found to be tender on palpation and swollen without any evidence of erythema. She had ultrasound scan of her breast which demonstrated a small amount of homogenous fluid encompassing her breast implant (see figures 4 and 5). She was prescribed Amoxicillin-Clavulanate 875-125 mg twice per day; nevertheless, she re-manifested three days later with worsening pain, swelling, as well as new erythema. She was taken to theatre where her breast abscess was drained with a total 200 MLS of purulent fluid and her breast implant was removed. Culture of her breast abscess grew Salmonella serogroup C. Based upon the sensitivity pattern of the cultured organism, her antibiotics was changed to sulfamethoxazoletrimethoprim 800-160 mg twice per day for 14 days and it was recommended that 4 additional months should pass before a new breast implant is inserted. Hall et al. [19] made the ensuing statements:

- $\quad$ Patients need to be counselled with regard to the potential for the development of haematogenous seeding of the breast cavity and implant following severe illness as well as bacteraemia.

- It might be reasonable to provide patients who have breast implants who are travelling to areas at a high-risk for traveller's diarrhoea or areas with limited medical resources with an antibiotic to take if they develop moderate to severe symptoms of traveller's diarrhoea while they were away on vacation. 


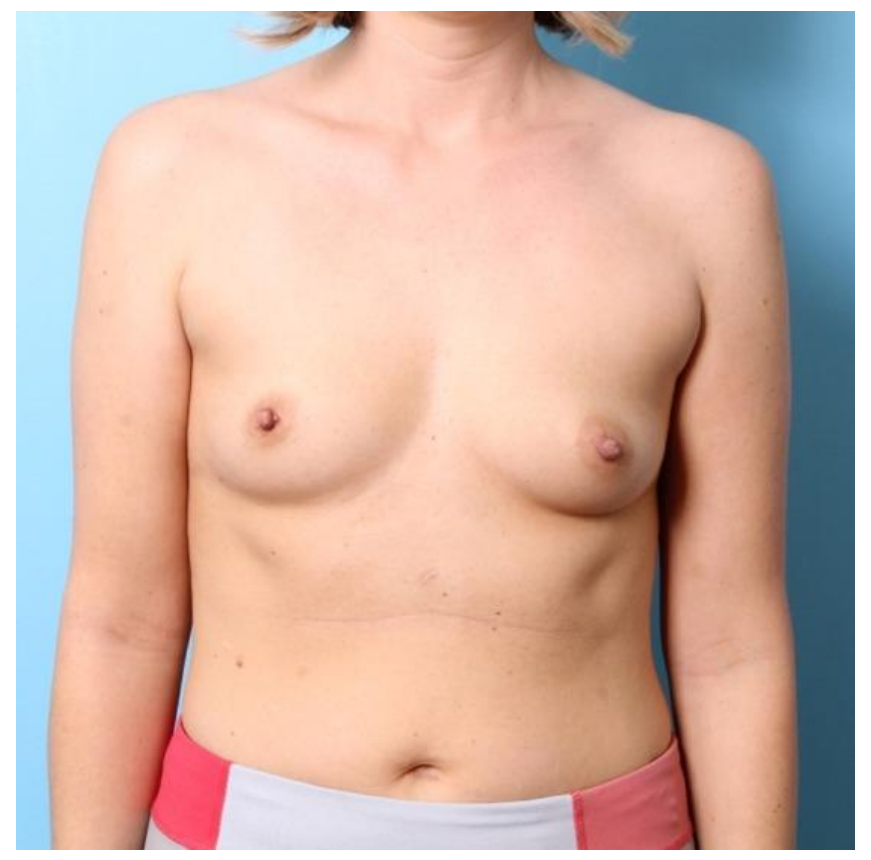

Figure 2: Preoperative image of patient's breasts demonstrating hypomastia asymmetric areolar location. Reproduced from: [19] Hall BR, Billue KL, Sanders SE, Meyer BR, Johnson PJ. Salmonella infection of breast implant associated with traveler's diarrhea: A case report. JPRAS Open. 2018 Sep 19;18:59-64. doi: 10.1016/j.jpra.2018.08.004. PMID: 32158838; PMCID: PMC7061617. https://pubmed.ncbi.nlm.nih.gov/32158838/under Copyright (C) 2018 The Authors This is an open access article under the CC BY-NC-ND license (http://creativecommons.org/licenses/by-nc-nd/4.0/).

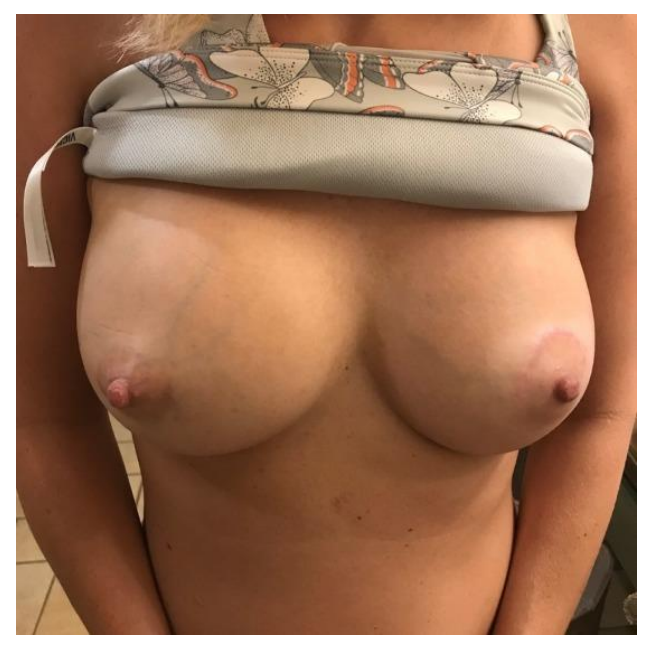

Figure 3: Image taken after having recovered from gastroenteritis at the time when the patient noticed tenderness and swelling of the right breast. Reproduced from: [19] Hall BR, Billue KL, Sanders SE, Meyer BR, Johnson PJ. Salmonella infection of breast implant associated with traveler's diarrhea: A case report. JPRAS Open. 2018 Sep 19;18:59-64. doi: 10.1016/j.jpra.2018.08.004. PMID: 32158838; PMCID: PMC7061617. https://pubmed.ncbi.nlm.nih.gov/32158838/ under Copyright (C 2018 The Authors This is an open access article under the CC BY-NC-ND license (http://creativecommons.org/licenses/by-nc-nd/4.0/). 


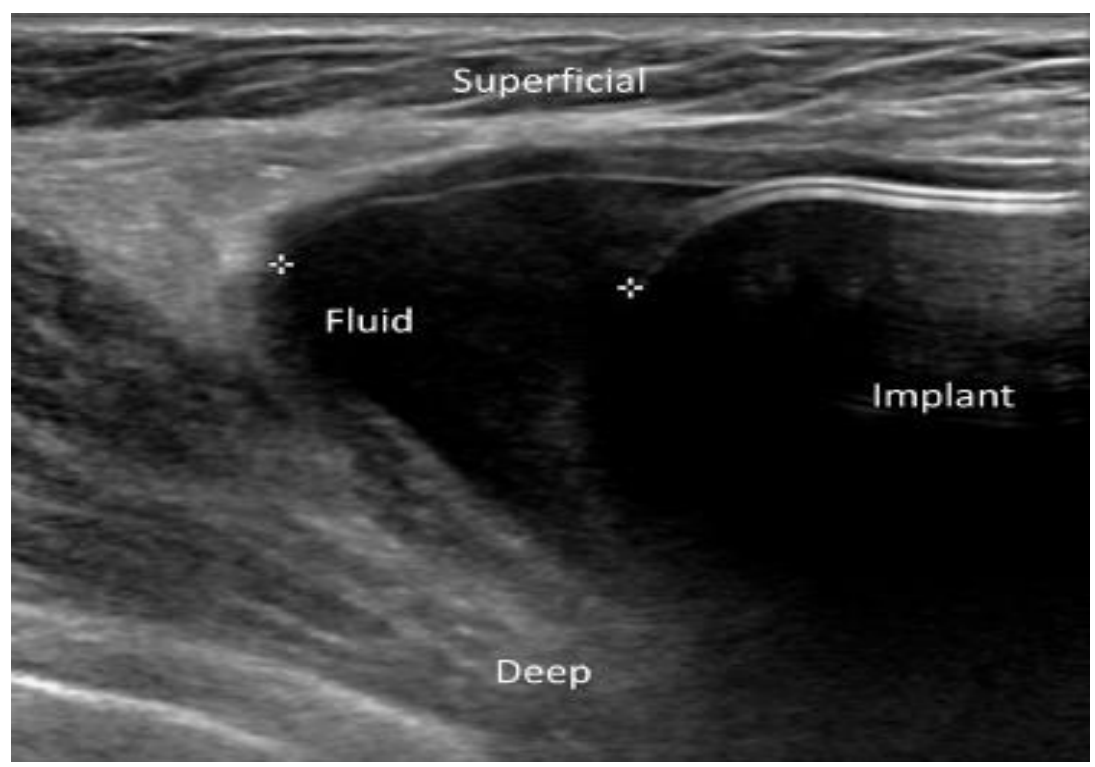

Figure 4: Ultrasound image demonstrating a small collection homogenous fluid at the inferior and ateral aspect of the breast outside of her implant at the 7 o'clock position. Reproduced from: [19] Hall BR, Billue KL, Sanders SE, Meyer BR, Johnson PJ. Salmonella infection of breast implant associated with traveler's diarrhea: A case report. JPRAS Open. 2018 Sep 19;18:59-64. doi: 10.1016/j.jpra.2018.08.004. PMID: 32158838; PMCID: PMC7061617. https://pubmed.ncbi.nlm.nih.gov/32158838/under Copyright $@ 2018$ The Authors This is an open access article under the CC BYNC-ND license (http://creativecommons.org/licenses/by-nc-nd/4.0/).

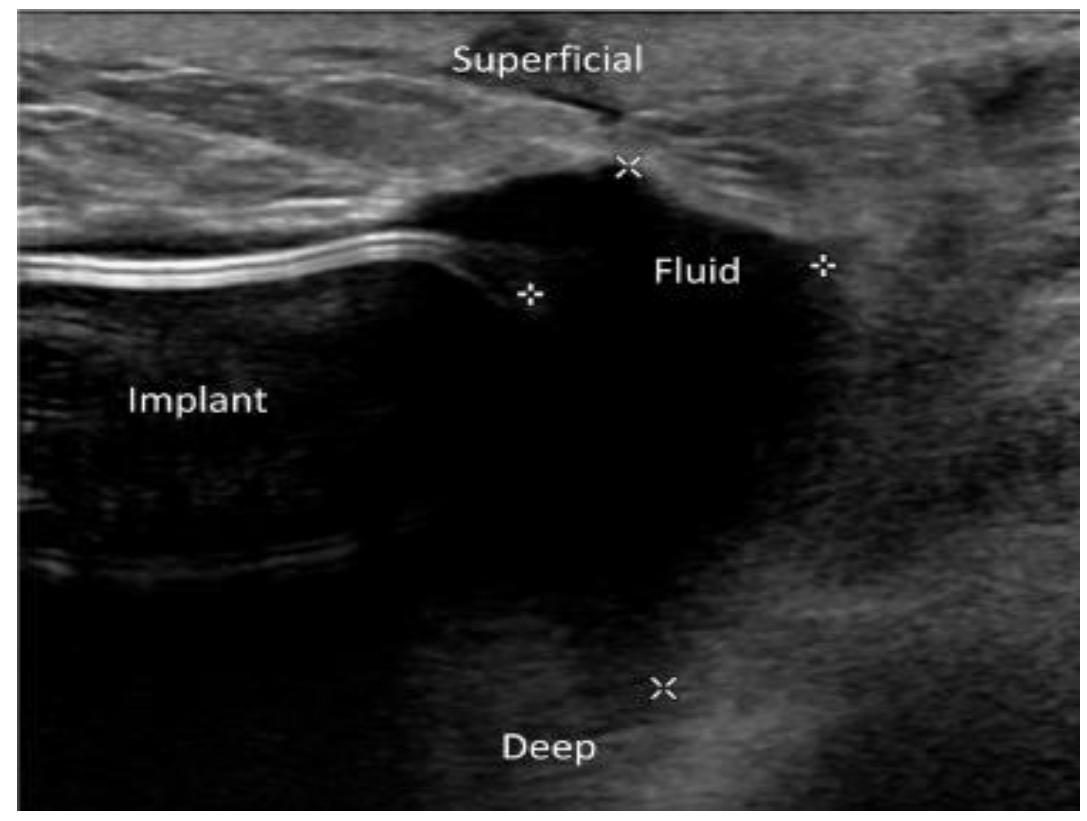

Figure 5: Ultrasound image demonstrating a small collection of homogenous fluid at the superior and lateral pole of the breast outside of her implant at the 11 o'clock position. Reproduced from: [19] Hall BR, Billue KL, Sanders SE, Meyer BR, Johnson PJ. Salmonella infection of breast implant associated with traveler's diarrhea: A case report. JPRAS Open. 2018 Sep 19;18:59-64. doi: 10.1016/j.jpra.2018.08.004. PMID: 32158838; PMCID: PMC7061617. https://pubmed.ncbi.nlm.nih.gov/32158838/ under Copyright (C 2018 The Authors This is an open access article under the CC BYNC-ND license (http://creativecommons.org/licenses/by-nc-nd/4.0/).

Deshpande et al [20]. reported a 35-year-old lady who had breast abscess that was caused by Salmonella Para-typhi A without any clinical evidence of having any enteric fever-like symptoms. The breast abscess case was managed with utilization of a combination antibiotic therapy and surgical option in order to treat the aetiological cause of the disease as well as the focal infection. Lessons that need to be learnt from this short summation include the following:
- The fact that Salmonella abscess of the breast could be encountered in individuals who have not had any evidence of past or present enteric fever or salmonella infection of any other organ and hence a high-index of suspicion for Salmonella infection should be on the mind of all clinicians who encounter cases of breast infection / abscess in all patients especially in individuals who are not lactating in that Staphylococcal abscess of the breast tends to be more common in lactating individuals. 
- Non-lactating individual females who are clinically found to have breast abscess should have Widal test as well as all individuals who have breast abscess should have pus/abscess fluid sent for microbiology culture and sensitivity tests.

- Clinicians should have a high-index of Salmonella abscess as a differential diagnosis of individuals who have breast abscess and they should also remember to ascertain in their history taking if the individual has a past history of enteric fever or diarrhoea and if they did then they should also ascertain if a definite diagnosis was made as well as if the patients had had treatment of the disease as well as the type of antibiotic they received with the inclusion of the duration of the treatment.

Brnčić et al [3]. reported a 70-year-old man who was known to have noninsulin dependent diabetes Mellitus, who had manifested with a swelling within his left breast over the preceding 9 months. He did complain of minimal pain in view of the fact that the mass had grown slowly over the 9 months period. His clinical examination demonstrated a $4 \mathrm{~cm} \mathrm{x} 4 \mathrm{~cm}$ left breast mass that was slightly painful with livid discoloration of the overlying skin of the breast. The nipple of the left breast was also noted to be retracted. He had mammography which demonstrated within the left retro-mammary pre-pectoral space, presence of two irregular, soft-tissue density masses which had micro-calcifications as well as calcifications which was suggestive of a possible malignancy, or less likely a breast abscess. The result of his tumour marker CA 15-3, was found to be 8.7 U/ML, which was within the normal range of the laboratory of between 0.1 and 31.3 U/ML. Upon diagnostic aspiration of the left breast mass, 20 millilitres of pus was obtained and cytological examination of the pus whilst awaiting the results of the microbiology culture and sensitivity results demonstrated features of suppurative inflammation. He underwent sub-cutaneous mastectomy of his left breast and histopathology examination of the left breast specimen demonstrated features of chronic inflammation without any features to suggest the possibility of malignancy. Microbiology examination and culture of the pus showed a growth of Salmonella enterica serotype Enteritidis that was sensitive to Amoxicillin-Clavulanic acid, ceftriaxone, trimethoprimsulfamethoxazole, as well as ciprofloxacin. He received ciprofloxacin oral medication. Pursuant to the establishment of Salmonella abscess of his left breast, his past medical history was reviewed and this revealed that he had developed an episode of severe diarrhoea and vomiting that lasted 5 days which was ensued by him developing anuria 2 days preceding his admission to the Infectious Diseases Department of one regional hospital. Upon his admission into the Infectious Diseases Department of the regional hospital, he was found to be severely dehydrated, with signs of acute renal failure, because of which he underwent haemodialysis 5 times that led to full recovery of his renal function. At that time Salmonella Enteritidis was isolated from his stool cultures. He did not receive any antibiotic treatment during this episode of Salmonella Enteritidis past infection. Some of the summating iterations made by Brnčić et al. [3] include the following:

- Abscesses of the breast usually tend to be caused by Staphylococcus Aureus and they tend to occur mainly during pregnancy and lactation, perhaps as a result of trauma of the nipple as well as intraductal stasis as postulated by Edelstein $[2]$.

- Associated symptoms of salmonella breast abscess usually tend to comprise of fever, chills, as well as varying local changes.

- It has been iterated that typhoid breast abscesses had been reported in up to $0.9 \%$ of cases of generalized related to Salmonella Enterica Serovar Typhi [2,4].

- Non-typhoidal salmonellae had been reported only sporadically as the causative organism of breast abscess [2,7,21]
- It has been documented that majority of patients who had been diagnosed as having non-typhoidal salmonella infection, tend to have self-limited gastroenteritis, and approximately $3 \%$ to $8 \%$ of the patients tend to develop secondary bacteraemia [22]

- It has also been stated that localized infections do develop in about $5 \%$ to $10 \%$ in individuals who develop non-typhoidal salmonella bacteraemia [23]

- With regard to other patients, particularly, those patients who are severely immunocompromised, primary non-typhoidal Salmonella bacteraemia, does occur, in the absence of preexisting signs of gastroenteritis and this also tends to be associated with a high incidence of extra-gastrointestinal organ involvement by the disease which has been reported in $34 \%$ of cases [22].

- It has been documented that focal extra-intestinal infections from non-typhoidal Salmonella had increased with regard to incidence during the preceding 2 decades [24]

- It has been iterated that extra-intestinal salmonellosis does occur more often in individuals who have underlying chronic conditions as well as immune deficiency $[25,26]$.

- It has been stated that risk factors for the development of focal infections that are caused by Salmonellas including salmonella breast abscess do include the ensuing: [25,26,27]

$$
\begin{array}{cl}
\circ & \text { Extremes of ages. } \\
\circ & \text { Immunosuppression. } \\
\circ & \text { Underlying malignancy. } \\
\circ & \text { Intravenous abuse of drugs. } \\
\circ & \text { As well as previous trauma. }
\end{array}
$$

Some of the lessons that need to be learnt from this case report include:

- Salmonella Enterica Serotype Enteritidis breast abscess could develop in a male therefore clinicians need to be vigilant and to have a high index of suspicion for the disease.

- $\quad$ Salmonella Enterica Serotype Enteritidis breast abscess could develop in individuals who have non-insulin dependent diabetes mellitus.

- Salmonella Enterica Serotype Enteritidis breast abscess could manifest in some cases with symptoms related to the breast without any knowledge of a previous Salmonella infection but after the diagnosis has been obtained, a review of the past medical history may reveal a past history of salmonella infection and it may also demonstrate that the Salmonella infection had not been treated previously even though the diagnosis had been made.

Sood [28] reported a 37-year-old lady who presented with fever on and off as well as pain within her right breast over the preceding 15 days. She had also noticed a lump within her right breast over the preceding 4 days. She had a one year history of type 2 diabetes mellitus with poor control of her glucose levels as well as a history of hypothyroidism. She was found to have a $4 \mathrm{~cm} \mathrm{x} 4 \mathrm{~cm}$ lump within her right breast on the lateral outer quadrant as well as she had bilaterally mobile lymph nodes. She also had fever with a temperature of 101.7 degrees F. The results of all her routine haematology and biochemistry blood tests were normal with the exception of the fact that her serum thyroid stimulating hormone (TSH) level was raised at $6.15 \mathrm{micro}$ IU/ML (normal range (0.2 to 4.20 micro IU/ML), and her fasting sugar (FBS) was raised at $130 \mathrm{MG} / \mathrm{DL}$ (normal range 74 to $99 \mathrm{MG} / \mathrm{DL}$ ). She had bilateral mammograms which did not demonstrate any abnormality. She had ultrasound scan of her abdomen which demonstrated mild enlargement of liver with fatty infiltration of the liver. She had ultrasound scan of her breast and axilla which revealed well-defined heterogeneously defined hypo-echoic mass lesion that measured about $3.6 \mathrm{~cm} \times 1.8 \mathrm{~cm}$ within her right breast which had involved the lateral aspect of the right breast. The dilated ducts did 
measure about 7-8 $\mathrm{mm}$ in calibre which showed thick echogenic material within it. The mammary ducts were seen to be covering towards the nipple. Many variable lymph nodes were seen in both axillary regions with the largest lymph node measuring $1.7 \mathrm{~cm} \times 1.3 \mathrm{~cm}$ showing evidence of central necrosis. Ultrasound-guided fine needle aspiration cytology (FNAC) was undertaken and the examination of the specimen showed many neutrophils against a necrotic background that was suggestive of breast abscess. She underwent ultrasound scan-guided drainage of the breast abscess and the drained pus was sent for sent for culture and sensitivity and she was given intravenous Amikacin 500 mg 12 hourly plus Clindamycin $600 \mathrm{mg}$ intravenously 12 hourly as well as Ofloxacin which she had been commenced on earlier was stopped.. The microbiology culture of the pus grew Salmonella Para-typhi A that was sensitive to Ceftriaxone, Chloramphenicol, Ampicillin, Tetracycline, Cotrimoxazole, Ciprofloxacin, as well as Ofloxacin but it was resistant to Nalidixic acid. Based upon the results of the pus culture sensitivity pattern, her treatment was changed to Ceftriaxone 2 grams intravenously 12 hourly. She had a WIDAL test and blood culture and both results were negative. She had a repeat ultrasound scan of her breast 3 days later and the scan did show thick pus within many dilated ducts in the lower outer quadrant of the breast with reactive lymph nodes. She underwent a repeat ultrasound-scan-guided aspiration drainage of the breast abscess and the pus which was sent for culture grew Salmonella Para-typhi A. She was discharged following 7 days of admission on Ceftriaxone 2 grams intravenously twice per day, Amikacin $250 \mathrm{mg}$ intravenously twice per day, and capsule Dalacin (Clindamycin) $300 \mathrm{mg}$ orally three times per day for 4 days and she was planned to be reviewed after 4 days. At her second follow-up 10 days after her discharge from hospital her abscess had completely resolved. A lesson learnt from this case report is that Salmonella breast abscess can be effectively treated by utilization of ultrasound scan-guided aspiration drainages and appropriate combination antibiotics based upon the sensitivity pattern of the cultured organism.

Singh et al [9]. reported a 29-year-old lady who had manifested with a lump within both of her breasts over the preceding 7 days that was associated with pain. The lumps were stated by her to be around her nipples and they were tender to touch with the overlying skin being warm. The pain was stated to be progressive in nature, sharp, as well as shooting pain. She had been having intermittent discharge of pus-like substance from her nipple which was milky with regard to its consistency. She did have high fever of 39 degrees centigrade over the preceding 1.5 months, which was improved by her intake of antipyretic medicament. She did have rare episodes of loose stools/ bowel motions over the preceding 1.5 months. Two weeks preceding the onset of her breast pain and breast lump, she did visit the medical out patients department of a hospital because of fever and she was empirically commenced on AmoxicillinClavulanic acid $625 \mathrm{mg}$ twice per day for 7 days; nevertheless, her fever did not resolve. Although she did have blood culture which grew Salmonella typhi that was resistant to amoxicillin, she did not return for follow-up and she did not therefore, receive an appropriate antibiotic treatment based upon the sensitivity pattern of the cultured organism. She had two children but she was not pregnant and was not lactating. Her clinical examination demonstrated a centrally located lump within her left breast just underneath her areola that measured $3 \mathrm{~cm} \mathrm{x} 3 \mathrm{~cm}$. She was also found to have a palpable axillary lymph node that measured about $2 \mathrm{~cm}$ on the left side. She had drainage of pus from both of her breast lumps which upon culture grew Salmonella typhi in both cases that was sensitive to chloramphenicol, cefotaxime, ciprofloxacin, and co-trimoxazole, but which was resistant to Ampicillin and Nalidixic acid. She did have a positive blood culture of Salmonella typhi 2 weeks earlier which had a similar antibiotic sensitivity pattern. The result of her WIDAL test was negative and the result of her routine haematology and biochemistry blood tests were within normal range with the exception of her serum glutamine oxaloacetic transaminases (SGOT) as well as serum glutamine pyruvic transaminase (SGPT) that were raised at 96.59 and $113.78 \mathrm{U} / \mathrm{L}$, respectively. She was commenced on oral ciprofloxacin $500 \mathrm{mg}$ twice per day for 2 weeks and she did respond favourably with regression of the lesions. Her stool and urine cultures were negative for Salmonella typhi during her manifestation and subsequent follow-up visits. Singh et al [9]. Iterated the following:

- Salmonella bacteraemia tends occasionally to be associated with extra-intestinal disease. [26]

- Salmonella typhi is capable of forming abscesses within a variety of organs including the liver, sub-cutaneous tissues, muscle and the skin.

- Bilateral abscesses of the breast due to Salmonella typhi are uncommon manifestations. [4]

- $\quad$ Their reported case of bilateral Salmonella breast abscess was associated with a detectable bacteraemia in the past.

Some of the lessons that need to be learnt from this case report include:

- Bilateral Salmonella breast abscesses could be encountered unexpectedly and this would require a high index of suspicion and it would require ensuring that pus from both breast abscesses should be submitted for culture and sensitivity.

- The finding of unilateral or bilateral salmonella breast abscess(es) could be associated with:

(a) Normal WIDAL test results (negative test).

(b) Normal haematology blood tests results as well as normal CRP.

(c) Negative stool and urine culture during the time of manifestation of the salmonella breast test.

(d) A previously non-appropriate treatment of a past episode of gastrointestinal salmonella infection if the salmonella organism is resistant to the antibiotic given or if no antibiotic was given for the treatment of a diagnosed Salmonella infection of the gastrointestinal tract organs.

Singh et al [4]. reported a case of bilateral breast abscess due to Salmonella typhi in an unmarried 35-year-old woman, who did not have any predisposing conditions for the development of Salmonella breast abscess. She manifested with fever, and painful swelling of both of her breasts. Salmonella typhi was cultured from both of her breast abscesses. Singh et al [4]. stated that such uncommon cause of breast abscess should be suspected with regard to females who do not have any evidence of predisposing factors for the development of Salmonella breast abscess in order to provide effective treatment of the disease.

Hiyam et al [29]. reported an unusual case of recurrent bilateral breast abscess which had been caused by multiple organisms in an immunocompetent non-lactating patient. They reported that a 41 -year-old non-diabetic Saudi single lady who had manifested in October 2015 with progressive left breast pain over the preceding 15 days that was associated with a lump within her left breast. She was found following thorough assessment to have a left breast abscess which was treated by means of intravenous antibiotics and incision and drainage of the breast abscess. Culture of the pus that was drained grew Pseudomonas aeruginosa (see table 1). Between October 2015 and January 2018 he required treatment for breast abscesses which had involved the breast which had involved the left breast alone, the right breast alone, or both breasts contemporaneously. In all including the first breast abscess the lady had developed breast abscesses on 10 different occasions between October 2015 and January 2018. The cultured bacteria did vary from one infection to the next and the organisms that were cultured would be summarised as 
follows: [1] October 2015 Left Breast -E Coli / Pseudomonas aeruginosa [2]. November 2015 Right and Left Breasts - E Coli / Proteus mirabilis [3]. January 2016 Right breast - E Coli / Pseudomonas aeruginosa [4] September 2016 Left Breast - E Coli / Proteus Mirabilis [5] September 2016 Right Breast -E Coli / Kocuria ristinae / Salmonella. [6] July 2017 - Right and Left Breast Mixed organisms [7]. July 2017 - Left and Right Breast -Proteus Mirabilis [8]. August 2017 - Left Breast -Proteus Mirabilis [9]. November 2017 - Right Breast - E Coli / Proteus Mirabilis, [10] January 2018 Right Breast - Klebsiella Pneumoniae / Citrobacter Species. She was investigated fully for the decrease in her appetite over the period between October 2015 and January 2018 and nothing was found except for positive Helicobacter Pylori stool antigen. Tests for hepatitis profile, HIV test, and tuberculosis (TB) test, were all negative as well as cancer screening test, $\operatorname{IgG}, \operatorname{IgM}$, IgE, were all normal. Anti endomyral antibodies were negative. Her breast biopsy was negative for cancer and for chronic inflammatory conditions [29].

A lesson that has been learnt from this case report is the fact that every pus that is drained from a breast abscess must be sent for culture and sensitivity including the primary breast abscess pus and all recurrent pusses that are drained because the causative organism and the antibiotic sensitivity pattern could be different. In the reported case there was a growth of Salmonella in one of the specimens contemporaneously with other bacteria.

\begin{tabular}{|l|l|l|}
\hline Time & Side of Breast & $\begin{array}{l}\text { Cultured organism from breast } \\
\text { abscess }\end{array}$ \\
\hline October 2015 & Left Breast & E Coli / Pseudomonas aeruginosa. \\
\hline November 2015 & Right and Left Breasts & E Coli / Proteus Mirabilis. \\
\hline January 2016 & Right Breast & E Coli / Pseudomonas aeruginosa. \\
\hline September 2016 & Left Breast & E Coli / Proteus Mirabilis \\
\hline September 2016 & Right Breast & E Coli / Kocuria ristinae / Salmonella \\
\hline July 2017 & Right and Left Breast & Mixed organism \\
\hline July 2017 & Left and Right Breast & Proteus Mirabilis \\
\hline August 2017 & Left Breast & Proteus Mirabilis \\
\hline November 2017 & Right Breast & E Coli / Proteus Mirabilis \\
\hline January 2018 & Right Breast & $\begin{array}{l}\text { Klebsiella Pneumoniae / Citrobacter } \\
\text { Species. }\end{array}$ \\
\hline
\end{tabular}

Table 1: From October 2015 till January 2018 patient admitted ten times with repeated bilateral breast abscess. Reproduced from: [29] Hiyam A H,

Hend A S, Majila A A. Recurrent bilateral Breast Abscess in Immunocompetent Women: A Case Report and Literature Review. Open Access J Surg 2018; 8(4): 555745. DOI: 1019080/OAJS.2018.08.555745 https://juniperpublishers.com/oajs/pdf/OAJS.MS.10.555745.pdf. https://juniperpublishers.com/oajs/pdf/OAJS.MS.ID.555745.pdf

\section{Conclusions}

- Salmonella breast abscess / mastitis is a rare disease which clinicians should have a high index of suspicion for.

- An antecedent history of previous enteric fever with a history of abdominal pain, diarrhoea, vomiting and fever does help in suspecting the disease which at times does need to be distinguished from carcinoma of the breast.

- The diagnosis tends to be confirmed by obtaining the culture results of the pus from the breast abscess / breast tissue.

- If all breast lesions are biopsied especially in cases of suspicion of a breast cancer and a past history of abdominal pain, diarrhoea and vomiting the results of the pathology examination of the specimen would negate malignancy and confirm an infection which would help in avoiding mastectomy procedures and enable utilization of antibiotics and drainage/aspiration of abscess to provide adequate treatment.

\section{Conflict of interest - None}

\section{Acknowledgements}

Acknowledgements to:

- Int J Cur Res Rev for granting permission for reproduction of figures and contents of their journal article.

- JPRAS Open. For granting permission for reproduction of figures and contents of their journal article under Copyright (C) 2018 The Authors This is an open access article under the CC BY-NC-ND license (http://creativecommons.org/licenses/by$\underline{\text { nc-nd/4.0/). }}$.
- Open Access Journal of surgery for granting copyright permission for reproduction of contents, figures, and tables from their previously published article.

\section{References}

1. Baran, I., Aksu, N. \& Aksoy, A. (2016) Breast abscess due to Salmonella Typhimurium in a patient with rheumatoid arthritis: a case report. BMC Infect Dis. 16: 348 .

2. Edelstein H. (1993) Breast abscess due to Salmonella serogroup $\mathrm{B}$, serotype Reading, in a young nonpuerperal woman. Clin Infect Dis.17(5):951-952. doi: 10.1093/clinids/17.5.951. PMID: 8286662.

3. Brncic N, Gorup L, Strcic M, Abram M, Mustac E. (2011) Breast abscess in a man due to Salmonella enterica serotype Enteritidis. J Clin Microbiol. 2012 Jan;50(1):192-193. doi: 10.1128/JCM.05361-11. Epub PMID: 22031702; PMCID: PMC3256715.

4. Singh S, Pandya Y, Rathod J, Trivedi S. Bilateral breast abscess: a rare complication of enteric fever. Indian J Med Microbiol.27(1):69-70. PMID: 19172067.

5. Banu A, Hassan MM, Anand M. (2013) Breast abscess: sole manifestation of Salmonella typhi infection. Indian J Med Microbiol. 31(1):94-5. doi: 10.4103/0255-0857.108753. PMID: 23508445.

6. Fernando S, Molland JG, Gottlieb T. Failure of oral antibiotic therapy, including azithromycin, in the treatment of a recurrent breast abscess caused by Salmonella enterica serotype Paratyphi A. Pathog Glob Health.106(6):366-9. doi: 10.1179/2047773212Y.0000000010. PMID: 23182142; PMCID: PMC4005136.

7. Al Benwan K, Al Mulla A, Izumiya H, Albert MJ. (2010) Erythema nodosum and bilateral breast abscesses due to 
Salmonella enterica serotype Poona. J Clin Microbiol. 48(10):3786-7. doi: 10.1128/JCM.00780-10. Epub 2010 Aug 11. PMID: 20702664; PMCID: PMC2953116.

8. Weisenberg E. (2021) Salmonella (typhoid and nontyphoidal). PathologyOutlines.

9. Singh G, Dasgupta M, Gautam V, Behera A, Ray P. (2011) Bilateral Breast Abscesses due to Salmonella Enterica Serotype Typhi. J Glob Infect Dis. 3(4):402-4. doi: 10.4103/0974777X.91069. PMID: 22224009; PMCID: PMC3250001

10. Barrett GS, MacDermot J. (1972) Breast abscess: a rare presentation of typhoid. Br Med J. 10;2(5814):628-9. doi: 10.1136/bmj.2.5814.628. PMID: 5031689; PMCID: PMC1788380.

11. Delori M, Abgueguen P, Chennebault JM, Pichard E, Fanello S. (2007) Breast abscess with Salmonella typhi and review of the literature. J Gynecol Obstet Biol Reprod (Paris) 36:709-12

12. Mahajan RK, Duggal S, Chande DS, Duggal N, Hans C, Chaudhry R. (2007) Salmonella enterica serotype Typhi from a case of breast abscess. J Commun Dis. 39(3):201-204. PMID: 18697587.

13. Razeq and associates [Razeq JH, Glenn A, Thomas G, Sholes A. (2000) First human case of Salmonella enterica serotype Landwasser recovered from breast fluid. J Clin Microbiol. 38(11):4300. doi: 10.1128/JCM.38.11.4300-4300.2000. PMID: 11185066; PMCID: PMC87593.

14. Al-Ishak Z, Sidkar O, Dobie D, Sircar T. (2018) Salmonella Enteritidis breast abscess: an unusual cause of breast abscess in the UK. BMJ Case Reports 2018:bcr-2018-226075

15. Agrawal S, Yadav VS, Srivastava A, Kapil A, Dhawan B. Breast abscess due to Salmonella paratyphi A : Case reports with review of literature. Intractable Rare Dis Res.7(2):130133. doi: 10.5582/irdr.2018.01031. PMID: 29862156; PMCID: PMC5982621.

16. Viswanathan R, Shah A H, Nagori L F, Gupta M K. (2015) Salmonella Typhi in Breast Abscess Bombay Hospital Journal. 57(03)

17. Seah X F V, Ngeow J H A, Thoon K C. (2015) Infant with Invasive Nontyphoidal Salmonellosis and Mastitis. Journal of Medical Sciences and Health

18. Murugesan N. Alagar Samy R, Viswanathan M S, Anbazhagan A. (2016) A Rare Case of Breast Abscess Due To Salmonella Typhi. Int J Cur Res Rev 8(5): 10 - 12.

19. Hall BR, Billue KL, Sanders SE, Meyer BR, Johnson PJ. (2018) Salmonella infection of breast implant associated with traveler's diarrhea: A case report. JPRAS Open. 18:59-64. doi: 10.1016/j.jpra.2018.08.004. PMID: 32158838; PMCID: PMC7061617.
20. Deshpande A, Dash L, Pandya JS, Zende M. Salmonella paratyphi A infection presenting as breast abscess. BMJ Case Rep. 12(4):e228887. doi: 10.1136/bcr2018-228887. PMID: 30962213; PMCID: PMC6453335.

21. Cheng S P, Liu C L, Chang Y C. (2006) Extraintestinal Salmonella infection appearing assuspicious breast mass. J. Ultrasound Med. 25:277-279

22. Shimoni Z, Pitlik S, Leibovici L, Samra Z, Konigsberger H, Drucker M, Agmon V, Ashkenazi S, Weinberger M. (1999) Nontyphoid Salmonella bacteremia: age-related differences in clinical presentation, bacteriology, and outcome. Clin Infect Dis. 28(4):822-7. doi: 10.1086/515186. PMID: 10825045.

23. Pegues D A, Miller S I. (2010) Salmonella species, including Salmonella Typhi, p 28872903. In Mandell GL, Bennett JE, Dolin R (ed), Mandell, Douglas, and Bennett's principles and practice of infectious diseases, 7th ed. Churchill Livingstone Elsevier, Philadelphia, PA

24. Moraitou E, Karydis I, Nikita D, Falagas ME. (2007) Case report: parotid abscess due to Salmonella enterica serovar Enteritidis in an immunocompetent adult. Int J Med Microbiol. 297(2):123-6. doi: 10.1016/j.ijmm.2006.11.005. Epub 2007 Jan 17. PMID: 17234452.

25. Ispahani P, Slack RC. (2000) Enteric fever and other extraintestinal salmonellosis in University Hospital, Nottingham, UK, between 1980 and 1997. Eur J Clin Microbiol Infect Dis.19(9):679-87. doi: 10.1007/s100960000341. PMID: 11057501.

26. Rodríguez M, de Diego I, Mendoza MC. Extraintestinal salmonellosis in a general hospital (1991 to 1996): relationships between Salmonella genomic groups and clinical presentations.J ClinMicrobiol. 36(11):3291-6. doi: 10.1128/JCM.36.11.3291-3296.1998. PMID: 9774581; PMCID: PMC105317.

27. Girardin F, Mezger N, Hächler H, Bovier PA. (2006) Salmonella serovar Give: an unusual pathogen causing splenic abscess. Eur J Clin Microbiol Infect Dis.25(4):2724.doi:10.1007/s10096-006-0122-2.PMID:16565825.

28. Sood S. (2015) Breast Abscess by Salmonella Paratyphi A: Case Report and Literature Review. Journal of Clinical and Diagnostic Research : JCDR.9(9):DD03-4.

29. Hiyam A H, Hend A S, Majila A A. (2018) Recurrent bilateral Breast Abscess in Immunocompetent Women: A Case Report and Literature Review. Open Access J Surg 8(4): 555745. DOI: 1019080/OAJS.2018.08.555745 